The authors suggest that the current recommendation of dissecting six lymph nodes in order to determine nodal involvement in patients with esophageal cancer should be amended to a higher threshold and that calculation of the ratio of positive lymph nodes to the number of nodes sampled could add prognostic information for patients classified as pN1.

Original article Bogoevski D et al. (2008) Is it time for a new TNM classification in esophageal carcinoma? Ann Surg 247: 633-641

\section{Imiquimod 5\% cream treats vulvar intraepithelial neoplasia effectively}

Surgical treatment of vulvar intraepithelial neoplasia (VIN) can cause disfigurement of the vulva and does not always prevent recurrence or progression because the most common cause of these lesions, human papillomavirus (HPV), is not eliminated. A less-invasive and more-effective treatment is, therefore, required. To address this need, van Seters et al. investigated the efficacy of a topical formulation of imiquimod, an immune-response modifier that is known to promote apoptosis in tumor cells.

A total of 52 patients (age range 22-71 years) with predominantly grade $2-3 \mathrm{VIN}$ were randomly assigned to either imiquimod $5 \%$ or placebo cream. Both creams were self-administered to lesions twice weekly for 16 weeks. HPVpositive lesions were detected in 25 patients in each group at baseline. Lesion size was measured every 4 weeks and biopsies were performed at baseline and at 20 weeks.

At the end of the study period, $21(81 \%)$ of 26 patients treated with imiquimod achieved a $>25 \%$ reduction in lesion size; none of the patients in the placebo group reached this end point. In addition, a complete response (100\% reduction in lesion size) was observed in 9 of the imiquimod-treated patients, all of whom remained disease-free at 12 months' follow-up. HPV was eradicated in 15 lesions treated with imiquimod but in only 2 placebo-treated lesions $(P<0.001)$. Regression of histological grade was observed in 18 patients treated with imiquimod, compared with 1 patient in the placebo group. Imiquimod treatment also provided notable relief of itching and pain over the duration of the study and follow-up period. The authors recommend imiquimod $5 \%$ cream as first-line therapy for patients with VIN.

Original article van Seters M et al. (2008) Treatment of vulvar intraepithelial neoplasia with topical imiquimod. N Engl J Med 358: 1465-1473

\section{Molecular markers prognostic for biochemical failure in prostate cancer}

Although there are established prognostic markers for prostate cancer, such as the Gleason score and serum concentration of prostate-specific antigen, these markers do not accurately predict the variation in outcome seen in patients with this disease. Vergis et al. evaluated molecular markers of hypoxia and angiogenesis that might better predict outcome in prostate cancer.

Biopsy samples were obtained from 201 patients with localized prostate cancer before they underwent radical radiotherapy as part of one of two prospective, randomized trials in the UK, and from 289 patients with localized prostate cancer who had undergone radical prostatectomy in a hospital in Denmark. The biopsy samples were immunohistochemically stained for VEGF, hypoxia inducible factor $1 a$ (HIF-1 $\alpha$ ) and osteopontin. Multivariate analysis showed that in the surgery cohort, increased VEGF, HIF-1 $\alpha$ and osteopontin expression each significantly predicted decreased freedom from biochemical failure $(P<0.0001$ for VEGF and HIF-1a; $P=0.0005$ for osteopontin); however, in the radiotherapy cohort, only increased VEGF and HIF-1 $1 \alpha$ expression were significant predictors of poor outcome $(P=0.008$ and $P=0.02$, respectively). In both cohorts, the molecular markers were prognostic for outcome independent of the Gleason score and initial prostate-specific antigen concentration.

The authors suggest that these markers might help to identify high-risk patients, for whom standard therapy is not sufficient, and who might be suitable for enrollment into clinical trials investigating more-aggressive treatment.

Original article Vergis R et al. (2008) Intrinsic markers of tumour hypoxia and angiogenesis in localised prostate cancer and outcome of radical treatment: a retrospective analysis of two randomised radiotherapy trials and one surgical cohort study. Lancet Oncol 9: 342-351 